\title{
Pressão arterial invasiva: conhecimento teórico dos profissionais de enfermagem de uma unidade de terapia intensiva adulto
}

\author{
Invasive blood pressure: theoretical knowledge of nursing professionals from an adult \\ intensive care unit
}

\section{Presión arterial invasiva: conocimientos teóricos del profesional de enfermería en una unidad de cuidados intensivos para adultos}

Roberta Paolli de Paiva Oliveira ${ }^{1 *}$, José Rocha Gouveia Neto², Mônica Gusmão Lafrande Alves ${ }^{2}$, Kelle Karolina Ariane Ferreira Alves ${ }^{3}$, Yane Camila Ferreira Guedes ${ }^{4}$, Aísha Sthéfany Silva de Meneses $^{5}$, Jennyfer Barros Sousa ${ }^{2}$, Andreza Layanne Bezerra dos Anjos ${ }^{6}$, Gleybson Felipe Marinho da Silva ${ }^{7}$, Taciana da Costa Farias Almeida 5 .

\section{RESUMO}

Objetivo: Avaliar o conhecimento dos profissionais de enfermagem que atuam em unidade de terapia intensiva de adultos sobre a medida da pressão arterial invasiva, e identificar a percepção dos profissionais quanto ao seu grau de conhecimento sobre o tema. Métodos: Estudo descritivo, transversal, quantitativo, realizado em um hospital público e de ensino. Aplicou-se um questionário, contendo dados de identificação, questões objetivas sobre a medida direta da pressão arterial e questões de auto avaliação com escala do tipo Likert. Os dados foram analisados a partir da abordagem quantitativa, utilizando o programa SSPS versão 21. Resultados: Constituíram a amostra 26 profissionais, dos quais sete $(26,9 \%)$ eram enfermeiros e $19(73,1 \%)$ técnicos de enfermagem. Os participantes apresentaram um número médio de 5-11 acertos, alcançando um desempenho regular. A maioria dos profissionais $(57,7 \%)$ expressaram-se pouco satisfeitos com o seu conhecimento, e em sua totalidade os sujeitos afirmaram necessitarem de capacitação. Conclusão: Existem lacunas no conhecimento teórico da equipe de enfermagem quanto a medida da pressão arterial invasiva, sendo necessário a implementação de medidas que busquem capacitar continuamente. A capacitação destes profissionais proporcionará um cuidado intensivo seguro e capaz de atender as necessidades urgentes de cuidado para esta clientela.

Palavras-chave: Unidades de terapia intensiva, Determinação da pressão arterial, Profissionais de enfermagem, Conhecimento.

\section{ABSTRACT}

Objective: Evaluate the knowledge of nursing professionals working in the intensive care unit of adults on the measurement of invasive blood pressure, and identify the perception of professionals regarding their degree of knowledge on the topic. Methods: Descriptive, transversal, quantitative study, carried out in a public and teaching hospital. A questionnaire was applied, containing identification data, objective questions about direct blood pressure measurement and self-assessment questions with a Likert scale. The data were analyzed using the quantitative approach, using the SSPS version 21 program. Results: The sample comprised 26 professionals, of whom seven (26.9\%) were nurses and 19 (73.1\%) nursing technicians. The participants had an average number of 5-11 hits, achieving a regular performance. Most professionals (57.7\%) expressed little satisfaction with their knowledge, and in their totality, the subjects said they needed training. Conclusion: There are gaps in the theoretical knowledge of the nursing team regarding the measurement of invasive blood pressure, requiring the implementation of measures that seek to continuously train. The training of these professionals will provide intensive care that is safe and capable of meeting the urgent care needs of this clientele.

Keywords: Intensive care units, Blood pressure determination, Nurse practitioners, Knowledge.

\footnotetext{
${ }^{1}$ Universidade Federal do Rio Grande do Norte (UFRN), Hospital Universitário Onofre Lopes (HUOP), Natal - RN. ${ }^{*}$ E-mail: roberta.paolli@gmail.com

${ }^{2}$ Universidade Federal de Campina Grande (UFCG), Campina Grande - PB.

${ }^{3}$ Faculdade Irecê (FAI), Irecê - BA.

${ }^{4}$ Centro Integrado de Tecnologia e Pesquisa-Faculdade Nossa Senhora de Lourdes, Campina Grande - PB.

${ }^{5}$ Universidade Federal da Paraíba (UFPB), João Pessoa - PB.

${ }^{6}$ Centro Universitário UNIFACISA, Campina Grande - PB.

${ }^{7}$ Centro de Ensino Avançar (INESP FACULDADES), Campina Grande - PB.
} 


\section{RESUMEN}

Objetivo: Evaluar el conocimiento de los profesionales de enfermería que laboran en la unidad de cuidados intensivos de adultos sobre la medición de la presión arterial invasiva, e identificar la percepción de los profesionales sobre su nivel de conocimiento sobre el tema. Métodos: Estudio descriptivo, transversal, cuantitativo, realizado en un hospital público y docente. Se aplicó un cuestionario que contenía datos de identificación, preguntas objetivas sobre medición directa de la presión arterial y preguntas de autoevaluación con escala Likert. Los datos fueron analizados con enfoque cuantitativo, utilizando el programa SSPS versión 21. Resultados: La muestra estuvo compuesta por 26 profesionales, de los cuales siete $(26,9 \%)$ eran enfermeros y $19(73,1 \%)$ técnicos de enfermería. Los participantes tuvieron un promedio de 5-11 aciertos, logrando un desempeño regular. La mayoría de los profesionales $(57,7 \%)$ expresaron poca satisfacción con sus conocimientos y, en su conjunto, los sujetos dijeron necesitar formación. Conclusión: Existen vacíos en los conocimientos teóricos del equipo de enfermería en cuanto a la medición de la presión arterial invasiva, requiriendo la implementación de medidas que busquen capacitarse continuamente. La formación de estos profesionales proporcionará cuidados intensivos seguros y capaces de cubrir las necesidades de atención urgente de esta clientela.

Palabras clave: Unidades de cuidados intensivos, Determinación de la presión sanguínea, Enfermeras practicantes, Conocimiento.

\section{INTRODUÇÃO}

Os pacientes clinicamente graves internos em Unidade de Terapia Intensiva (UTI) requerem cuidados especializados, suporte e Monitorização Hemodinâmica (MH) contínuos (VENTURI V, et al., 2016). A MH é o elemento fundamental para avaliar a evolução clínica dos pacientes críticos (DIAS FS, et al., 2014). Por meio dela é possível avaliar variáveis hemodinâmicas importantes como o fluxo sanguíneo, a pré-carga cardíaca e a resistência vascular periférica, informações fundamentais que viabilizam a otimização das terapias adotadas, orientam as intervenções e permitem a rápida tomada de decisão (VENTURI V, et al., 2016; RILEY LE, et al., 2017; SAUGEL B, et al., 2018).

No que se refere aos métodos utilizados na $\mathrm{MH}$, a mensuração da Pressão Arterial (PA) ganha destaque pela importância para o paciente crítico. Na prática clínica da UTI, as mensurações da PA são usualmente obtidas de forma indireta e direta. O método indireto é considerado mais prático e é realizado de forma não invasiva e intermitente, utilizando a técnica oscilométrica. Já o método direto mensura a PA de modo contínuo e invasivo através de um cateter arterial periférico (SAUGEL B, et al., 2014; NAIK BI e DURIEUX ME, 2014).

A medida da PA por meio de cateteres arteriais periféricos é considerada padrão ouro no monitoramento da PA em pacientes críticos, uma vez que este método proporciona valores acurados da PA e ritmo cardíaco (RILEY LE, et al., 2017). Nos quadros clínicos em que o paciente apresenta extremos de níveis pressóricos e ritmos cardíacos irregulares, como na fibrilação atrial, ou está no intraoperatório e pós-operatório de cirurgias de grande porte, a monitorização da PA através do método indireto se torna menos confiável (SAUGEL B, et al., 2014; STERGIOU GS, et al., 2012; LEHMAN LW, et al., 2013). Portanto, os pacientes que apresentam instabilidade hemodinâmica e que estão em uso de vasopressores, devem ser monitorizados através do método contínuo da Pressão Arterial Invasiva (PAI) (NAIK BI e DURIEUX ME, 2014; RHODES A, et al., 2017).

Para mensurar a PAI e obter dados sobre a pressão arterial sistêmica sistólica, diastólica e média, é necessário a inserção de um cateter em uma artéria periférica por meio de punção percutânea. Este cateter é conectado a um equipo preenchido com solução salina que tem por função conectar diretamente o sistema da artéria ao transdutor de pressão, que por sua vez deverá captar as oscilações de pressões detectadas pela linha arterial, transformando-as em sinais elétricos, que serão transmitidos para um monitor, onde poderão ser visualizadas sob a forma de ondas de PA. Os locais disponíveis para a colocação do cateter compreendem a artéria radial, braquial, femoral e axilar, sendo a artéria radial comumente mais utilizada, devido aos baixos índices de complicações e ao seu fácil acesso (NAIK BI e DURIEUX ME, 2014; DING XR, et al., 2016; KIM WY, et al., 2013).

A inserção de um cateter arterial também possibilita a obtenção de amostras de sangue arterial para análise do equilíbrio ácido-base e outros exames. Embora as taxas de complicações relacionadas a PAI sejam baixas, este método de avaliação da PA pode gerar respostas adversas, como isquemia, infecção, hemorragia e trombose (RILEY LE, et al., 2017; SAUGEL B, et al., 2014). 
No entanto, deve-se reconhecer que ambos os métodos utilizados em UTI (direto e indireto) possuem limitações inerentes e diferentes níveis de complexidade. Segundo Almeida TCF e Lamas JLT (2013), existem falhas no conhecimento dos enfermeiros quanto a medida direta da PA, o que pode interferir na veracidade dos dados obtidos e nas condutas deste profissional.

Desta forma, cabe a equipe interprofissional do cuidado intensivo compreender os princípios básicos das tecnologias e as necessidades do paciente, com o propósito de decidir pela melhor técnica e mensurar de forma fidedigna a PA, evitando o tratamento impróprio a partir da obtenção de valores equivocados (SAUGEL B, et al., 2014; NAIK BI e DURIEUX ME, 2014; KIM WY, et al., 2013).

Diante do exposto, a pergunta norteadora do estudo foi: qual o conhecimento teórico que os profissionais de enfermagem de uma UTI de adultos apresentam sobre a medida da PA realizada com o método direto? Assim, este estudo propôs avaliar o conhecimento dos profissionais de enfermagem que atuam em Unidade de Terapia Intensiva de adultos sobre a medida da pressão arterial invasiva, e identificar a percepção dos profissionais quanto ao seu grau de conhecimento sobre o tema.

\section{MÉTODOS}

Trata-se de um estudo descritivo, transversal, com abordagem quantitativa, realizado em uma UTI adulto de um hospital público e de ensino, localizado em um município do interior do estado da Paraíba. O estudo foi realizado em uma UTI de médio porte, composta por dez leitos clínicos. Este local foi escolhido para o estudo por ser campo de prática para discentes de graduação em enfermagem, e realizar atendimento no âmbito do Sistema Único de Saúde (SUS), sendo também a unidade de referência em terapia intensiva para o município e região.

Constituíram a amostra vinte e seis profissionais de enfermagem, sendo sete enfermeiros e dezenove técnicos de enfermagem. A amostragem foi do tipo não probabilística por conveniência. Adotaram-se como critérios de inclusão: ser enfermeiro ou técnico de enfermagem atuante na UTI, independentemente do tempo de formação e atuação; estar presente no setor no momento da coleta dos dados. E como critérios de exclusão: enfermeiros e técnicos de enfermagem que estivessem de férias ou licença médica durante 0 período de coleta de dados.

Os dados foram coletados no período de janeiro a março de 2018, por meio de um questionário adaptado e validado, composto por três partes (ALMEIDA TCF e LAMAS JLT, 2013). A parte 1: oito quesitos com dados de identificação; parte 2: dez questões objetivas sobre a medida da PAI, com cinco alternativas, sendo apenas uma delas correta, de acordo com a literatura estudada. Para o tratamento dessas questões, aquelas que não apresentaram marcação ou que apresentaram mais de uma alternativa marcada, foram consideradas na análise, como não sabe a resposta ou a resposta está incorreta; e parte 3: seis questões objetivas, sendo uma delas com escala do tipo Likert, destinadas à auto avaliação dos profissionais de enfermagem quanto ao seu conhecimento sobre PAI. Também foi solicitado que eles avaliassem a importância do assunto e a necessidade de capacitação sobre o mesmo em seu ambiente de trabalho e justificativas sobre a necessidade de treinamento.

No procedimento de coleta de dados, a pesquisadora comparecia ao setor no início dos plantões (manhã, tarde e noite), e convidava o profissional a responder o questionário. O profissional recebia um envelope, contendo o Termo de Consentimento Livre e Esclarecido (TCLE) em duas vias, uma cópia do questionário, uma caneta e um adesivo autocolante para lacrar o envelope ao final.

Os participantes foram orientados a não comentar sobre o conteúdo do questionário com os demais membros da equipe, uma vez que se tratava de avaliação de conhecimento sobre procedimento específico do setor de trabalho. Esta orientação se tornou necessária para evitar vieses na pesquisa, uma vez que devido à rotina do setor, estes profissionais não poderiam ser remanejados para um local específico para respondêlo. A pesquisadora permanecia na instituição durante o tempo de preenchimento do questionário para retirar dúvidas quanto à interpretação das questões, caso ocorressem, e para recolher o instrumento ao final de cada turno. 
Os dados coletados foram codificados e digitados em planilha do Excel e posteriormente analisados a partir da abordagem quantitativa, utilizando o programa SSPS versão 21. Após análise, os resultados foram apresentados de forma descritiva e em tabelas, e discutidos a luz da literatura pertinente.

A pesquisa foi apreciada e aprovada pelo Comitê de Ética e Pesquisa, sob parecer no 2.459.339/2018, CAAE no 81147317.8.0000.5182, e realizada em conformidade com os pressupostos éticos da Resolução no 466/12 do Conselho Nacional de Saúde. Todos os participantes assinaram o TCLE.

\section{RESULTADOS}

Participaram do estudo 26 profissionais de enfermagem atuantes em UTI adulto. Destes, $21(80,8 \%)$ pertenciam ao sexo feminino. A idade dos profissionais variou de 31 a 52 , com uma média de $38,81( \pm 5,9)$ anos.

Quanto a categoria de atuação no setor, sete $(26,9 \%)$ eram enfermeiros e $19(73,1 \%)$ técnicos de enfermagem. O tempo de atuação do setor variou de nove meses a 31 anos, com uma média de 9,6 $( \pm 8,2)$ anos.

Em relação a formação acadêmica, 21 (80,8\%) possuíam curso superior, e cinco $(19,2 \%)$ curso técnico. Quatorze técnicos de enfermagem $(73,6 \%)$ possuíam nível superior. Em relação ao tipo de instituição de formação, 14 (53,8\%) obtiveram sua formação em instituição pública e $12(46,2 \%)$ em instituição privada.

Vinte e dois $(80,6 \%)$ afirmaram realizar a medida da PA invasiva no setor e quatro $(15,4 \%)$ não a realizavam. Vinte e seis (100\%) declararam não ter recebido treinamento do setor/instituição sobre a medida de PA invasiva. Vale salientar que a medida da PAl estava sendo realizada no setor desde junho de 2017. Após a aplicação do questionário, observaram-se os seguintes resultados apresentados a seguir (Tabela 1).

Tabela 1 - Distribuição do número e percentual de acertos e erros no teste de conhecimento sobre a medida da PAI. $n=26$.

\begin{tabular}{llc}
\multicolumn{1}{c}{ Questões } & $\begin{array}{c}\text { Acertos } \\
\mathbf{N}(\%)\end{array}$ & $\begin{array}{c}\text { Erros } \\
\mathbf{N}(\%)\end{array}$ \\
\hline $\begin{array}{l}\text { 1. A pressão adequada a ser aplicada na bolsa } \\
\text { pressurizada do sistema de monitorização. }\end{array}$ & $18(69,2 \%)$ & $08(30,8 \%)$ \\
2. A melhor curva da PAS no monitor. & $16(61,5 \%)$ & $10(38,5 \%)$ \\
$\begin{array}{l}\text { 3. A melhor curva da PAD no monitor. } \\
\text { 4. Qual onda representava a medida direta da PA em } \\
\text { punção de artéria femoral }\end{array}$ & $16(61,5 \%)$ & $10(38,5 \%)$ \\
$\begin{array}{l}\text { 5. A ordem de prioridade para punções de artéria } \\
\text { percutânea. }\end{array}$ & $18(69,2 \%)$ & $22(84,6 \%)$ \\
6. Qual a alteração de curva exibida no monitor. & $08(30,8 \%)$ & $18(30,8 \%)$ \\
7. Qual o tempo de troca do cateter percutâneo e o & $13(50,0 \%)$ & $13(50,0 \%)$ \\
sistema de monitorização. & $16(61,5 \%)$ & $10(38,5 \%)$ \\
8. A definição do teste de Allen. & $11(42,3 \%)$ & $15(57,7 \%)$ \\
9. Principais complicações da cateterização periférica \\
arterial.
\end{tabular}

Fonte: Oliveira RPP, et al., 2021.

Os participantes apresentaram um número médio de 5,11 acertos (questões). Para cada questão foi atribuído um escore de $10 \%$ por acerto. Dezesseis profissionais (61,5\%) contabilizaram um escore de $50 \%$ ou mais acertos. Considerando o desempenho por categoria de atuação, sete enfermeiros (100\%) e nove técnicos de enfermagem (47,3\%) obtiveram escore de $50 \%$ ou mais acertos. 
Após responder ao questionário, os profissionais responderam a uma questão do tipo escala de Likert, quanto a sua satisfação em relação ao seu conhecimento sobre a medida da PAI. Nenhum participante se sentiu totalmente satisfeito. Três $(11,5 \%)$ consideram-se satisfeitos com o seu conhecimento sobre a temática, $15(57,7 \%)$ expressaram-se pouco satisfeitos, quatro $(15,4 \%)$ insatisfeitos e dois $(7,7 \%)$ totalmente insatisfeitos. Dois sujeitos $(7,7 \%)$ não responderam a esta questão. Ao relacionar o nível de satisfação e a média do número de acertos obtidos, observaram-se os seguintes resultados apresentados (Tabela 2).

Tabela 2 - Comparação do nível de satisfação em relação ao conhecimento sobre a medida da PAI e a média do número de acertos obtidos no questionário. $\mathrm{n}=26$.

\begin{tabular}{lccc}
\hline \multicolumn{1}{c}{ Nível de satisfação } & $\mathbf{N}(\%)$ & Escore superior a 50\% & Escore inferior a 50\% \\
\hline Satisfeito & $03(11,5 \%)$ & $02(66,7 \%)$ & $01(33,3 \%)$ \\
Pouco satisfeito & $15(57,7 \%)$ & $08(53,3 \%)$ & $07(46,7 \%)$ \\
Insatisfeito & $04(15,4 \%)$ & $03(75,0 \%)$ & $01(25,0 \%)$ \\
Totalmente insatisfeito & $02(7,7 \%)$ & $01(50,0 \%)$ & $01(50,0 \%)$ \\
Não respondeu & $02(7,7 \%)$ & - & - \\
\hline
\end{tabular}

Fonte: Oliveira RPP, et al., 2021.

Prosseguindo a auto avaliação, os profissionais atribuíram conceitos e notas ao seu conhecimento teórico e prático. Em relação ao conhecimento teórico, nenhum participante o considerou como ótimo, sete (26,9\%) conceituaram como bom, $10(38,5 \%)$ regular, seis $(23,1 \%)$ ruim e dois $(11,5 \%)$ péssimo. Quanto ao conhecimento prático, dois $(7,7 \%)$ consideraram como ótimo, $11(42,3 \%)$ consideraram como bom, sete $(26,9 \%)$ regular, cinco $(19,3 \%)$ ruim. Nenhum profissional atribuiu conceito péssimo ao seu conhecimento prático. Um $(3,8 \%)$ não respondeu a esta questão.

Quanto às notas atribuídas ao autoconhecimento teórico, houve uma variação de 0 a 9 pontos para 0 conhecimento teórico, com uma média de 5,65. Para o conhecimento prático, houve uma variação de 0 a 10 pontos, com uma média de 6,54.

As notas autoatribuídas foram comparadas aos conceitos autoatribuídos pelos sujeitos, estabelecendo-se as seguintes faixas de notas: ótimo $(8,0-10,0)$, bom $(7,0-7,9)$, regular $(5,0-6,9)$, ruim (3,0-4,9) e péssimo $(0,0-$ 2,9), conforme adotado em estudo realizado por Almeida TCF e Lamas JLT (2013). Em seguida, foi estabelecido uma relação entre as notas do desempenho no questionário e os conceitos autoatribuídos pelos sujeitos, conforme demonstrado a seguir (Tabela 3).

Tabela 3 - Distribuição de notas de desempenho no questionário e conceitos autoatribuídos pelos sujeitos acerca do conhecimento teórico sobre medida da PAI. $n=26$.

\begin{tabular}{lcccccc}
\hline \multirow{2}{*}{$\begin{array}{c}\text { Desempenho no teste } \\
\text { teórico }\end{array}$} & \multicolumn{5}{c}{$\begin{array}{c}\text { Conceito com base na nota autoatribuída ao } \\
\text { conhecimento teórico } \\
\mathbf{N}(\%)\end{array}$} & $\begin{array}{c}\text { Total } \\
\mathbf{N}(\%)\end{array}$ \\
\cline { 2 - 7 } & Ótimo & Bom & Regular & Ruim & Péssimo & \\
\hline Ótimo & 00 & $04(15,4)$ & $01(3,8)$ & 00 & 00 & $05(19,3)$ \\
\hline Bom & 00 & $01(3,8)$ & 00 & 00 & 00 & $01(3,8)$ \\
\hline Regular & $02(7,7)$ & $01(3,8)$ & $03(11,5)$ & $03(11,5)$ & $01(3,8)$ & $10(38,5)$ \\
\hline Ruim & $01(3,8)$ & $01(3,8)$ & $05(19,3)$ & 00 & 00 & $07(26,9)$ \\
\hline Péssimo & 00 & $01(3,8)$ & 00 & $01(3,8)$ & $01(3,8)$ & $03(11,5)$ \\
\hline Total & $03(11,5)$ & $08(30,8)$ & $09(34,6)$ & $04(15,4)$ & $02(7,7)$ & $26(100)$ \\
\hline
\end{tabular}

Fonte: Oliveira RPP, et al., 2021.

Ao relacionar as notas do desempenho teórico com o tipo de instituição de formação dos indivíduos, obteve-se os seguintes resultados apresentados (Tabela 4). 
Tabela 4 - Desempenho no teste teórico por tipo de instituição de formação. $n=26$.

\begin{tabular}{lccccc}
\hline \multirow{2}{*}{$\begin{array}{c}\text { Tipo de instituição de } \\
\text { formação }\end{array}$} & \multicolumn{5}{c}{ Desempenho no teste teórico } \\
& N (\%) \\
\cline { 2 - 6 } & Ótimo & Bom & Regular & Ruim & Péssimo \\
\hline Pública & $03(21,4)$ & $01(7,2)$ & $05(35,7)$ & $03(21,4)$ & $02(14,3)$ \\
Privada & $02(16,7)$ & 00 & $05(41,7)$ & $04(33,3)$ & $01(8,3)$ \\
\hline
\end{tabular}

Fonte: Oliveira RPP, et al., 2021.

Nesta amostra, não houve diferença entre o número de acertos e o tipo de instituição de formação, pois a mediana de acertos, assim como o conhecimento regular foi igual para ambas.

Ainda no contexto de auto avaliação, os participantes identificaram as questões que mais sentiram dificuldade para responder. Questões que tratavam sobre o reconhecimento da curva de pressão no monitor foram apontadas como as mais difíceis para serem respondidas, das quais, oito profissionais $(30,8 \%)$ referiram maior dificuldade para responder a segunda questão (A pressão sistólica nesta curva de pressão arterial está representada no ponto...), 10 (38,5\%) a terceira questão (A pressão diastólica nesta curva de pressão arterial está representada no ponto...), 15 (57,7\%) a quarta questão (A onda que melhor representa a medida direta da PA em punção de artéria femoral é...), $11(42,3 \%)$ a sexta questão (Ao observarmos a curva abaixo no monitor, podemos afirmar que...). Dois profissionais (7,7\%) afirmaram sentir dificuldade para responder todas as questões e um $(3,8 \%)$ declarou não sentir dificuldade para responder as questões.

Todos os participantes demonstraram interesse em se atualizar e afirmaram necessitar de capacitação sobre a medida da PAI. Dentre as justificativas apresentadas para a necessidade de capacitação no setor, pode-se destacar: renovar conhecimentos $(26,9 \%)$, conhecimento próprio $(23,0 \%)$, segurança do paciente $(15,3 \%)$, melhorar as práticas e técnicas $(15,3 \%)$, conhecimento teórico e prático insatisfatório $(15,3 \%)$, falta de conhecimento $(11,5 \%)$.

Quanto a nota relativa à importância desta temática no setor de trabalho, houve uma variação de 0 a 10 pontos, com uma média de $8,81.23$ profissionais ( $88,4 \%$ ) atribuíram notas entre $8-10$ ao serem questionados sobre a importância deste assunto para o setor. Ao relacionar a nota de importância (8-10) atribuída pela maioria $(88,4 \%)$ ao assunto para o setor com as notas de desempenho obtidas pelos sujeitos, nota-se que destes, quatro $(17,3 \%)$ obtiveram desempenho ótimo (8-10), um $(4,3 \%)$ desempenho bom $(7-7,9)$, nove $(39,1 \%)$ regular $(5-6,9)$, seis $(26,0 \%)$ ruim $(3-4,9)$ e três $(13,0 \%)$ desempenho péssimo $(0,0-2,9)$.

\section{DISCUSSÃO}

Observou-se entre os profissionais de enfermagem uma predominância do sexo feminino, caracterizando a feminilização da profissão, corroborando com os resultados de outro estudo que buscou identificar o perfil sociodemográfico de enfermeiros atuantes em UTI, assim como a média de idade 38,81 anos (VIANA RAPP, et al., 2014).

Quanto à formação acadêmica, nota-se o número expressivo de técnicos de enfermagem que possuíam nível superior $(73,6 \%)$, mesmo atuando como nível médio no setor. Estudo aponta um aumento expressivo na busca por cursos de graduação em enfermagem por parte dos auxiliares e técnicos, revelando o interesse em se capacitar, com a perspectiva de se estabelecer no mercado de trabalho em cargos de maior complexidade técnico-científica (MACHADO MH, et al., 2016).

Em sua totalidade, os profissionais declararam não ter recebido treinamento no setor/instituição sobre a medida da PAI, dado relevante, uma vez que $80,6 \%$ afirmaram realizar este procedimento no setor, e o mesmo ter sido instituído na rotina do setor há um ano. Este dado merece atenção, pois, para que se assegure o rigor técnico-cientifico necessário à monitorização da PAl, é fundamental o entendimento profundo do princípio de medição e dos critérios de qualidade da onda de pressão (SAUGEL B, et al., 2020).

No que se refere aos termos legais que envolvem a PAI, a resolução COFEN № 390/2011 reflete sobre a realização da punção arterial tanto para fins de gasometria como para monitorização da PAl como um 
procedimento privativo do enfermeiro no âmbito da equipe de enfermagem, reforçando a importância do desenvolvimento de competências e habilidades que busquem garantir o rigor técnico-científico aos procedimentos (COFEN, 2011).

Os dados apresentados na Tabela 1 revelam expressiva dificuldade dos profissionais em identificar a artéria através da análise da sua curvatura no monitor, apresentando $84,6 \%$ de erros, assim como, no reconhecimento da morfologia e causa de uma anormalidade de curva pressórica $(69,2 \%)$, na identificação correta das principais complicações da cateterização periférica arterial $(57,7 \%)$, e o tempo de troca do cateter percutâneo e do sistema de monitorização (50,0\%).

A avaliação criteriosa da curva de pressão e a compreensão da sua morfologia normal, são elementos necessários para entender o funcionamento do sistema de monitorização e detectar alterações nas condições do cateter arterial e do sistema de zeragem. É igualmente importante entender os efeitos da fisiologia e do local de medição, pois, estes fatores podem modificar a aparência morfológica da onda de pressão e interferir na sua interpretação (SAUGEL B, et al., 2014).

Uma revisão sistemática destacou a crescente evidência de infecções sanguíneas relacionadas a cateteres arteriais. Este estudo revela que a escolha da artéria radial sobre a femoral contribui para a redução do risco de infecção, o que torna a escolha da artéria e a prevenção e identificação desta complicação, fatores decisivos para o sucesso da monitorização da PAI (O'HORO JC, et al., 2014).

Em contrapartida, observa-se um desempenho razoável de acertos em questões como a identificação da pressão adequada a ser aplicada na bolsa pressurizada do sistema de monitorização $(69,2 \%)$, da PAS $(61,5 \%)$ e PAD $(61,5 \%)$ representadas na curva de pressão arterial, no reconhecimento da ordem de prioridade para punções de artéria percutânea $(69,2 \%)$, e na técnica correta de medida direta da PA $(53,8 \%)$.

A falta do ajuste ou o ajuste incorreto do zero hidrostático, assim como, o nivelamento incorreto do transdutor arterial e inconformidades na bolsa pressurizada, são fontes de medições incorretas da PAl, por isso, torna-se essencial a realização da técnica de medição correta a fim de evitar interpretações equivocadas sobre a condição clínica do paciente e o tratamento incorreto por falsos valores da PA (SAUGEL B, et al., 2014; NAIK BI e DURIEUX ME, 2014; AIRES MJ, et al., 2012; JACQ G, et al., 2015).

Assim, é crucial que os profissionais que manuseiam este sistema possuam conhecimento suficiente sobre o que está executando, afim de minimizar erros e complicações ao paciente crítico, especialmente no ambiente de UTI, local onde mais se realiza este procedimento técnico, e onde é imperativo o conhecimento adequado do profissional de enfermagem ao manusear este sistema de monitorização.

A literatura sobre o conhecimento dos enfermeiros no que se refere a monitorização hemodinâmica invasiva é limitada, como afirma um estudo realizado no Sudão com 90 enfermeiros. Os resultados deste estudo demonstraram que a maior parte dos enfermeiros estudados $(84,4 \%)$ não sabiam conceituar o teste de Allen (AHMED WAM, et al., 2016). Não tanto expressivo, mas relevante, visto a importância deste teste, o número de erros obtidos nesta questão foi de $38,5 \%$, dado que pode estar associado ao número pequeno da amostra deste estudo em comparação ao estudo realizado no Sudão (AHMED WAM, et al., 2016).

A média de acertos obtida no teste teórico $(5,11)$, foi semelhante à nota média que os indivíduos se auto conceituaram $(5,65)$, revelando que, após responder ao questionário, os profissionais tornaram-se conscientes do déficit de conhecimento que possuíam sobre a temática. Nota-se que, de acordo com a nota média obtida no teste de conhecimento, houve um desempenho regular, deficiente e com falhas relevantes, semelhante aos resultados apontados em outros estudos (ALMEIDA TCF e LAMAS JLT, 2013; AHMED WAM, et al., 2016). Este achado é preocupante, visto que, os profissionais considerados mais "aptos" a realizarem este procedimento de medida, apresentam lacunas importantes no conhecimento e reconhecem este déficit, reforçando que nunca tiveram treinamento sobre o assunto, mesmo com a técnica instituída recentemente na rotina do setor campo de estudo.

Observa-se na prática clínica, que muitos procedimentos técnicos de enfermagem são instituídos nas rotinas setoriais sem que os profissionais sejam treinados adequadamente para realizá-los com conhecimento e segurança, o que pode ocasionar falhas na assistência direta ao paciente, especialmente em setores intensivos. 
Quanto a satisfação em relação ao seu conhecimento, a maioria dos profissionais se consideraram pouco satisfeitos quanto ao seu conhecimento sobre a medida da PAI, ainda que $53,3 \%$ destes tenham alcançado um escore de acertos superior a $50 \%$, o que pode indicar dificuldade em relação a temática abordada ou insegurança quanto as respostas assinaladas, resultando na tomada de consciência do seu conhecimento deficiente. Isto pode ser visualizado nos dados que demonstram as questões que os profissionais sentiram mais dificuldade para responder, onde questões de desempenho razoável como a segunda $(30,8 \%)$ e a terceira $(38,5 \%)$ foram apontadas como as de maior dificuldade, seguidas da quarta $(57,7 \%)$ e sexta $(42,3 \%)$ questão.

Contudo, apesar da consciência demonstrada pela maioria dos participantes, existem aqueles que se auto conceituaram como ótimo/bom e apresentaram um desempenho regular no teste teórico, demonstrando uma falsa impressão em relação ao seu conhecimento sobre a temática em questão, uma vez que 10 (38,5\%) deles tiveram um desempenho regular (5,0-6,9) de acordo com o número de acertos, mas apenas três $(11,5 \%)$ se auto conceituaram como regular; outros dois $(7,7 \%)$ se auto conceituaram como ótimo, um $(3,8 \%)$ como bom, três $(11,5 \%)$ como ruim, e um $(3,8 \%)$ como péssimo.

Os profissionais, em sua totalidade, afirmaram necessitar de capacitação sobre o assunto, mostrando que foram capazes de reconhecer suas fragilidades e limitações quanto a medida da PAI, resultado semelhante foi evidenciado em estudo realizado na região sudeste do Brasil (ALMEIDA TCF e LAMAS JLT, 2013). Dentre as justificativas apresentadas, o conhecimento teórico e prático insatisfatório $(15,3 \%)$ e a falta de conhecimento $(11,5 \%)$ merecem atenção, pois revelam que o conhecimento adquirido durante a formação foi insuficiente, além de evidenciar a escassez de ações de educação continuada promovidas pelo serviço.

A maioria $(88,4 \%)$ atribuiu nota média de 8,81 para esta temática, considerado como um ponto positivo, pois demonstra que os profissionais são conscientes da importância deste assunto para a UTI e para os pacientes.

\section{CONCLUSÃO}

Este estudo identificou lacunas no conhecimento teórico dos enfermeiros e técnicos de enfermagem quanto a medida da PAI, assim como, o reconhecimento pelos profissionais deste déficit, fato preocupante que pode interferir nas condutas e cuidados prestados ao paciente crítico. Neste sentido, torna-se necessário a implementação de medidas que busquem capacitar continuamente os profissionais, tanto pelo desempenho regular apresentado pelos participantes, como pela expressiva afirmação da necessidade de capacitação e atualização. Contudo, este estudo possui limitações, o campo de estudo se trata da única instituição na região a realizar a medida da PAl, o que nos proporcionou um número reduzido como amostra, possibilitando um estudo apenas descritivo.

\section{REFERÊNCIAS}

1. AHMED WAM, et al. Invasive hemodynamic monitoring at critical care units in Sudan: assessment of nurses performance. J Health Spec, 2016; 4(3):196-201.

2. ALMEIDA TCF, LAMAS JLT. Nurses of adult intensive care unit: evaluation about direct and indirect blood pressure measurement. Rev Esc Enferm USP, 2013;47(2):369-376.

3. ARIES MJ, et al. Intra-arterial blood pressure reading in intensive care unit patients in the lateral position. $J$ Clin Nurs, 2012; $21(13-14): 1825-1830$.

4. CONSELHO FEDERAL DE ENFERMAGEM. Resolução n. 390/2011. Normatiza a execução, pelo enfermeiro, da punção arterial tanto para fins de gasometria como para monitorização de pressão arterial invasiva. Brasília: COFEN, 2011.

5. DIAS FS, et al. Hemodynamic monitoring in the intensive care unit: a Brazilian perspective. Rev Bras Ter Intensiva, $2014 ; 26(4): 360-366$.

6. DING XR, et al. Continuous blood pressure measurement from invasive to unobtrusive: celebration of 200th birth anniversary of Carl Ludwig. IEEE J Biomed Health Inform, 2016; 20(6):1455-1465.

7. JACQ G, et al. Modalities of invasive arterial pressure monitoring in critically ill patients: a prospective observational study. Medicine (Baltimore), 2015; 94(39): e1557.

8. KIM WY. Radial to femoral arterial blood pressure differences in septic shockpatients receiving high-dose norepinephrine therapy. Shock, 2013; 40(6): 527-531. 
9. LEHMAN LW, et al. Methods of blood pressure measurement in the ICU. Crit Care Med, 2013; 41(1): 34-40.

10. MACHADO MH, et al. Características gerais da enfermagem: o perfil sócio demográfico. Enferm Foco, 2016; 7(n.esp): 09-14.

11. NAIK BI, DURIEUX ME. Hemodynamic monitoring devices: putting it all together. Best Pract Res Clin Anaesthesiol, $2014 ; 28(4): 477-488$.

12. O'HORO JC, et al. Arterial catheters as a source of bloodstream infection: a systematic review and meta-analysis. Crit Care Med, 2014; 42(6):1334-1339.

13. RHODES A, et al. Surviving sepsis campaign: international guidelines for management of sepsis and septic shock: 2016. Intensive Care Med, 2017; 43(3):304-377.

14. RILEY LE, et al. Comparison of noninvasive blood pressure monitoring with invasive arterial pressure monitoring in medical ICU patients with septic shock. Blood Press Monit, 2017; 22(4):202-207.

15. SAUGEL B, et al. Measurement of blood pressure. Best Pract Res Clin Anaesthesiol, 2014; 28(4): $309-322$.

16. SAUGEL B, et al. Advanced hemodynamic monitoring in intensive care medicine: a German web-based survey study. Med Klin Intensivmed Notfmed, 2018; 113(3): 192-201.

17. SAUGEL B, et al. How to measure blood pressure using an arterial catheter: a systematic 5-step approach. Crit Care. 2020; 24(1):172.

18. STERGIOU GS, et al. Automated blood pressure measurement in atrial fibrillation: a systematic review and metaanalysis. J Hypertens, 2012; 30(11): 2074-2082.

19. VENTURI V, et al. O papel do enfermeiro no manejo da monitorização hemodinâmica em unidade de terapia intensiva. Rev Recien, 2016; 6(17):19-23.

20. VIANA RAPP, et al. Profile of an intensive care nurse in different regions of Brazil. Texto Contexto Enferm, 2014; 23(1):151-159. 